\title{
Ellenbogenarthroskopie: Zugänge, Anatomie, Komplikationen
}

\author{
Julian Dexel, Philip Kasten
}

\section{Zusammenfassung}

Die Ellenbogenarthroskopie hat sich zu einem sicheren und wichtigen Verfahren zur Diagnostik und Therapie von Ellenbogenpathologien entwickelt. $\mathrm{Zu}$ den etablierten Indikationen gehören die diagnostische Arthroskopie, die Entfernung freier Gelenkkörper, die Osteophytenabtragung, die Synovektomie, die Behandlung der Osteochondrosis dissecans und die Arthrolyse bei Kontrakturen des Ellenbogens. Aufgrund der Nähe der neurovaskulären Strukturen zu den Zugangsportalen sind eine genaue Kenntnis der Anatomie und arthroskopische Erfahrung notwendig. Die Lagerung, Markierung der Landmarken und die korrekte Platzierung der Zugänge sind Voraussetzung, um eine zielgerichtete, schnelle und komplikationsarme Operation durchführen zu können. Die Komplikationsrate ist verglichen mit Knieoder Schulterarthroskopien höher und liegt insgesamt bei ca. $10 \%$. Die gefürchteten Nervenläsionen sind meistens transient, treten sie aber permanent auf, haben sie weitreichende Folgen für die Patienten.

\section{Elbow Arthroscopy: Portal Place- ment, Anatomy, Complications}

Arthroscopy of the elbow has become a safe and reliable procedure for the diagnosis of and therapy for elbow disorders. Established indications include diagnostic arthroscopy, removal of loose bodies, removal of osteophytes, synovectomy, treatment of osteochondritis dissecans and capsular release. Especially the anatomic vicinity of the neurovascular structures to the portals requires a thorough knowledge of elbow anatomy and some experience with the procedure. The exact positioning of the patient, identification of the landmarks and knowledge of the correct portal placement are necessary to perform a safe and effective operation and to avoid complications. The overall complication rate with $10 \%$ is higher when compared to knee or shoulder arthroscopy. Nerve lesions are transient most of the time; however, when permanent they cause substantial problems for the patient.

beträgt in Flexion/Extension $145 / 0 / 5^{\circ}$ und Pro- und Supination 75/0/85 .

\begin{abstract}
Das Ellenbogengelenk ist ein Scharniergelenk mit einer Rotationsachse. Es setzt sich zusammen aus dem knöchern gut geführten Humeroulnargelenk, dem durch das Lig. anulare radii stabilisierte Humeroradialgelenk und dem proximalen Radioulnargelenk mit gemeinsamem Kapsel-Band-Apparat. Der normale Bewegungsumfang des Ellenbogengelenks
\end{abstract}

Anatomie

OP-JOURNAL 2012; 28: 66-71

(C) Georg Thieme Verlag KG Stuttgart · New York DOI http://dx.doi.org/10.1055/s-0031-1298485

Die meisten Alltagstätigkeiten erfordern einen Bewegungsumfang des Ellenbogens von Flexion/Extension 130/30/0 $\left(100^{\circ}\right)$, sowie einen Rotationsumfang von Pronation/Supination $50 / 0 / 50^{\circ}$ $\left(100^{\circ}\right)\left(, 100^{\circ}\right.$-Regel“ nach Morrey) [1].

Die Stabilität wird durch statische Komponenten (humeroulnare, humeroradiale und radioulnare Gelenkfläche, mediales und laterales Kollateralband, ventrale Kapsel, Membrana interossea, Lig. anulare) und dynamische Komponenten
(Mm. brachialis, biceps brachii, triceps brachii und anconeus) erreicht.

\section{Neurovaskuläre Strukturen}

Das Ellenbogengelenk ist in unmittelbarer Nachbarschaft umgeben von neurovaskulären Strukturen. Der N. ulnaris zieht dorsal durch den Sulcus nervi ulnaris und zwischen den oberflächlichen und tiefen Fingerbeugern nach distal. Die A. ulnaris verläuft ebenfalls dorsal zwischen oberflächlichen und tiefen Fingerbeugern nach distal [2].

Der N. medianus verläuft beugeseitig medial der A. brachialis und der Bizepssehne in die Fossa cubitalis. Der N. medianus verläuft medial zwischen den beiden Köpfen des M. pronator teres nach distal. Der N. radialis tritt zwischen dem M. brachioradialis und brachialis in die Ellenbeuge ein und teilt sich in einen Ramus superficialis und profundus. Der Ramus profundus des N. radialis tritt durch den M. supinator. Die Kubitalregion wird sensibel über die Nn. cutanei antebrachii medialis, lateralis und posterior versorgt. [3]

\section{Anästhesie}

Die Allgemeinanästhesie hat den Vorteil der Möglichkeit einer sofortigen postoperativen Beurteilung der neurovaskulären Strukturen, der vollständigen Muskelrelaxation, der freien Positionierung des Armes bei der Lagerung und der suffizienten Schmerzausschaltung bei angelegter Blutsperre.
Der Eingriff kann auch ohne Probleme in Regionalanästhesie bei wachem Patienten durchgeführt werden. Dies hat die bekannten Vorteile der Regionalanästhesie einschließlich der Spontanatmung, der Vermeidung eines Blutdruckabfalls oder postoperativer Übelkeit. Die Regionalanästhesie bietet die Option einer guten postoperativen Schmerztherapie über eine fortgesetzte Medikamenten- 


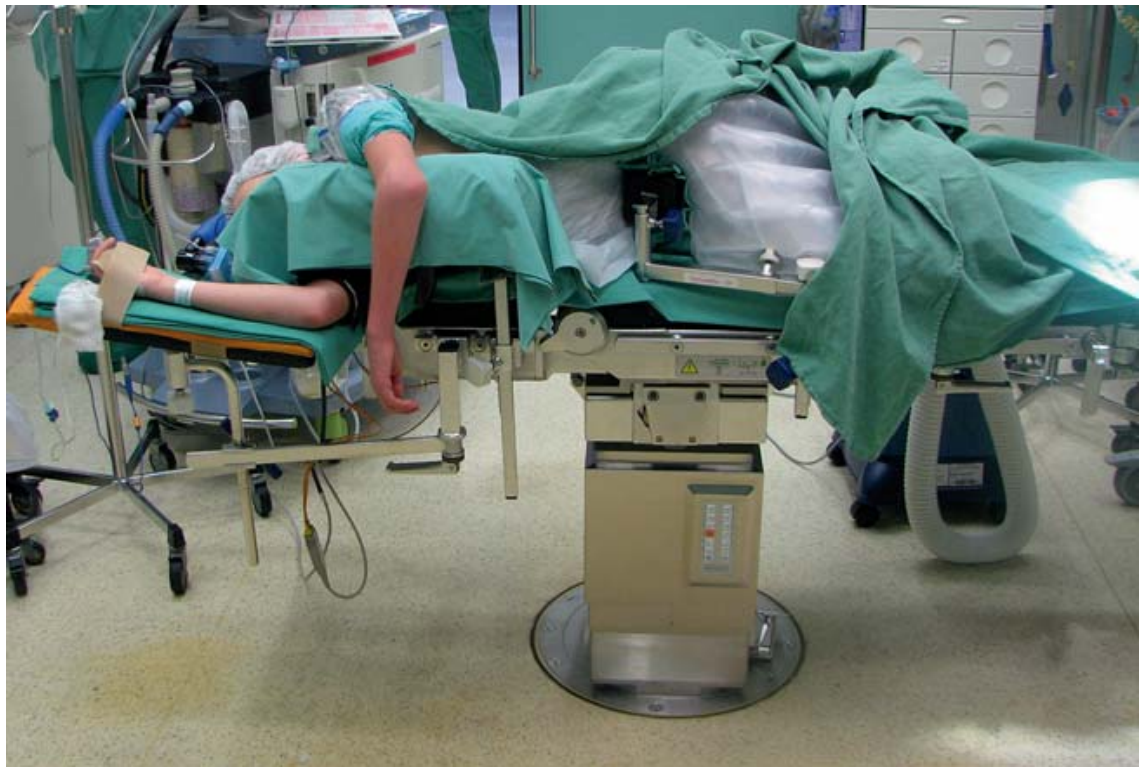

Abb. 1 Die Seitenlagerung bietet eine freie Lagerung des Ellenbogens und gute Zugangsmöglichkeiten für den Operateur und die Anästhesie.

applikation über Katheter, jedoch keine Möglichkeit einer sofortigen postoperativen Nervenbeurteilung. Eine Kombination von Allgemeinanästhesie mit postoperativer Kontrolle der Neurologie und anschließender regionalen Analgesie ist eine weitere Möglichkeit [4,5].

\section{Patientenlagerung}

Vor der definitiven Lagerung des Patienten in Narkose sollte eine seitenvergleichende Untersuchung beider Ellenbogengelenke in Rückenlage des Patienten erfolgen, um den Bewegungsumfang und die Bandstabilität zu beurteilen. Die Untersuchung ist am aussagekräftigsten, wenn sie vom Operateur sowohl vor der Narkose als auch in Narkose durchgeführt wird [6].

Es empfiehlt sich die Anlage einer Blutsperre, zur Verbesserung der Sicht in der Arthroskopie, mit einem Druck von ca. $80 \mathrm{mmHg}$ oberhalb des systolischen Blutdrucks (ca. $200 \mathrm{mmHg}$ ).

Es bestehen 3 Möglichkeiten der Lagerung des Patienten: in Rücken-, Seiten- oder Bauchlagerung [7-9]. Bei der Rückenlagerung, die zuerst für die Ellenbogenarthroskopie beschrieben wurde, ist der Ellenbogen $90^{\circ}$ gebeugt und der Arm an einem Extensionsgalgen fixiert [7]. Die Rückenlage hat den Vorteil eines einfachen anästhesiologischen Managements und eine für den Chirurgen gewohnte Anatomie. Das anteriore Kompartiment zeigt nach oben und das posteriore nach unten. Der Wechsel auf ein offenes Verfahren, wie eine mediale Kollateralbandrekonstruktion, ist leicht möglich. Nachteil der Lagerung ist der Zugang zum posterioren Kompartiment und die relative Instabilität durch die Fixation am Extensionsgalgen. Zudem ist die Sterilität während des Einhängens des Armes zu beachten.

In der Bauchlagerung, die zuerst von Poehling beschrieben wurde, befindet sich der zu operierende Arm seitlich auf einem speziellen Oberarmhalter oder einem kleinen Armhalter, auf den eine kleine Rolle befestigt ist [9]. Der Arm ist im Ellenbogengelenk $90^{\circ}$ flektiert und in der Schulter $90^{\circ}$ abduziert. Ein Extensionssystem wird nicht benötigt. Der Vorteil der Bauchlagerung ist, dass der Arm stabil gelagert ist und alle Standardzugänge erreicht werden können. Der Hauptnachteil ist der schlechte Zugang für das Atemwegsmanagement für die Anästhesie und die Problematik, dass die Operation schwer bei wachem Patienten in Regionalanästhesie durchgeführt werden kann. Ein weiterer Punkt ist, dass beim Desinfizieren der Haut der Arm recht stark in Retroversion und Abduktion gehalten werden muss, damit man den Arm an der Auflagefläche nicht unsteril macht.

Aus der von Poehling beschriebenen Bauchlage hat sich die Seitenlage entwickelt $[9,10]$. Erstmals wurde die Seitlagerung beschrieben als eine Methode, die dem Arm mehr Stabilität gibt als die
Rückenlage [8]. Der Arm ist stabil gelagert, und eine Extensionsvorrichtung wird nicht benötigt. Die Seitenlagerung bietet wie die Bauchlagerung gute $\mathrm{Zu}$ gangsmöglichkeiten zu allen Ellenbogengelenkskompartimenten. Des Weiteren kann der Ellenbogen frei gelagert werden, und es besteht Bewegungsfreiheit für die arthroskopischen Geräte. Die Operation kann ggf. auch offen fortgeführt werden. Die Atemwege sind für die Anästhesie zugänglich, und die Operation kann auch bei wachem Patienten in Regionalanästhesie erfolgen.

Der Patient wird ventral am Os pubis und dorsal am Sakrum und Thorax mit insgesamt 3 Seitstützen stabilisiert und der Arm von einem höhenverstellbaren gepolstertem Armbügel in $90^{\circ}$-Ellenbeugung gelagert [5]. Die Knöchel und das Fibulaköpfchen sollten mit Gelmatten weich gelagert werden. Unter den Thorax knapp unterhalb der Achsel sollte ein Schaumstoff- oder ein Gelkissen eingeschoben werden, um den Plexus zu entlasten. Der nicht operierte, unten liegende Arm sollte $90-100^{\circ}$ abgespreizt und $90^{\circ} \mathrm{im}$ Ellenbogen gebeugt kopfwärts gelagert werden, damit er den Operateur nicht stört (Abb. 1).

Nach erfolgter Lagerung sollte der Operateur die Instrumentenhandhabung durch verschiedene Portale simulieren, um eine Beeinträchtigung der Bewegungsfreiheit zu erkennen.

\section{Landmarken}

Nach Lagerung, Hautdesinfektion und sterilem Abdecken erfolgt die Markierung der Landmarken und Portale mit einem Hautmarkierungsstift. Der mediale Epikondylus ist prominenter als der laterale, und neben der Olekranonspitze sind diese 3 Strukturen gut palpabel. Das Radiusköpfchen ist durch eine Pround Supination distal des lateralen Epikondylus zu tasten.

Die Landmarken (Epicondylus medialis und lateralis, Olekranon, Radiusköpfchen und Capitulum humeri) und der Verlauf des N. ulnaris sowie die Portale werden angezeichnet (Abb. 2).

Nach Markierung der Landmarken und Aktivierung der Blutsperre erfolgt die Auffüllung des Gelenks mit Kochsalz. Hierzu wird der „soft spot“, im Zentrum eines gleichschenkligen Dreiecks, das durch den lateralen Epikondylus, das 


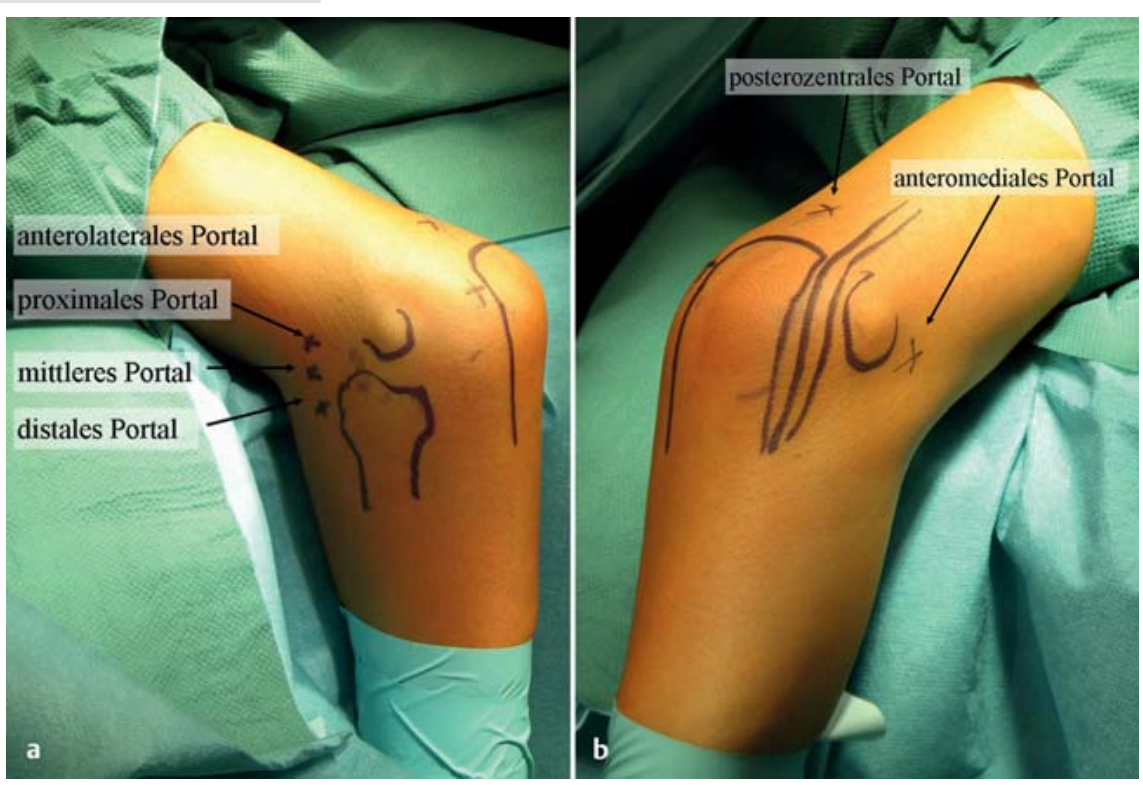

Abb. 2 a und b Die Landmarken zur Orientierung werden angezeichnet. a Epicondylus lateralis, Olekranon, Radiusköpfchen sowie das proximale, mittlere und distale anterolaterale Portal. b Markierung des Olekranons, des Epicondylus medialis und des Verlaufs des N. ulnaris sowie des posterolateralen, posterozentralen und des anteromedialen Portals.

Olekranon und das Radiusköpfchen gebildet wird, aufgesucht.

Es werden bei einer nicht geschädigten/ verdickten Kapsel ca. 20-30 ml Kochsalzlösung benötigt, um das Ellenbogengelenk aufzufüllen [11]. Hierdurch werden die neurovaskulären Strukturen vom Gelenk in die Peripherie verdrängt und die Verletzungsgefahr reduziert [12].

Insbesondere wird der $\mathrm{N}$. radialis nach ventral aus dem Bereich des radialen $\mathrm{Zu}$ gangs verdrängt. Nach dem Auffüllen breitet sich die Kapsel nach dorsolateral bogenförmig hinter dem radialen Epikondylus und um das Olekranon dorsal aus. Bei Flüssigkeitsaustritt aus der Kanüle kann von einer intraartikulären Lage ausgegangen werden (Abb. 3).

Der Arthroskopieturm sollte am gegenüberliegenden Fußende positioniert werden, damit die Schläuche und $\mathrm{Zu}-$ und Abflüsse über den Patienten oder einen Tisch zuverlässig fixiert werden können und eine gute Sicht auf den Monitor besteht.

\section{Portale}

Bei der Anlage der Portale ist immer die Regelposition des Ellenbogens $\left(90^{\circ}\right.$ Flexion und Mittelstellung zwischen Pround Supination) anzustreben, um eine Veränderung der Lagebeziehung der neurovaskulären Strukturen zu vermei- den. Es wird nur die Haut indiziert, subkutan erfolgt eine stumpfe Präparation, um sensible Hautnerven zu schützen.

Um geeignete Portale für die Ellenbogenarthroskopie zu benutzen, ist es wichtig, die Anatomie und die sicheren Zonen am Gelenk zu kennen. Man kann die Arthroskopie medial oder lateral beginnen [5].

\section{Anterolaterales Portal}

Dieses Portal sollte angelegt werden, wenn die knöchernen Landmarken noch gut zu tasten sind und bevor sich ein Schwellungszustand einstellt. Ursprünglich wurde das anterolaterale Portal $3 \mathrm{~cm}$ distal und $2 \mathrm{~cm}$ anterior des lateralen Kondylus, also unmittelbar anterior und proximal des Radiusköpfchens angelegt [7,13]. Aufgrund der Nähe des $\mathrm{N}$. radialis wurde dieser Zugang zugunsten einer weiter proximalen Anlage verlassen, die das Risiko einer Nervenverletzung minimiert [14]. Das mittlere anterolaterale Portal (ca. $1-2 \mathrm{~cm}$ ventral und $1,5-2 \mathrm{~cm}$ distal des Epicondylus radialis humeri) wird somit von vielen Chirurgen bevorzugt [15]. Dieser Zugang liegt zwischen dem Radiuskopf und dem ventralen Anteil des Capitulum. Als weitere Option besteht der proximale anterolaterale Zugang, der ca. $2 \mathrm{~cm}$ proximal und $1 \mathrm{~cm}$ ventral des Epicondylus radialis humeri liegt [14]. Es konnte in Studien gezeigt werden, dass der $\mathrm{N}$. radialis fast doppelt so weit von diesem Portal ent-

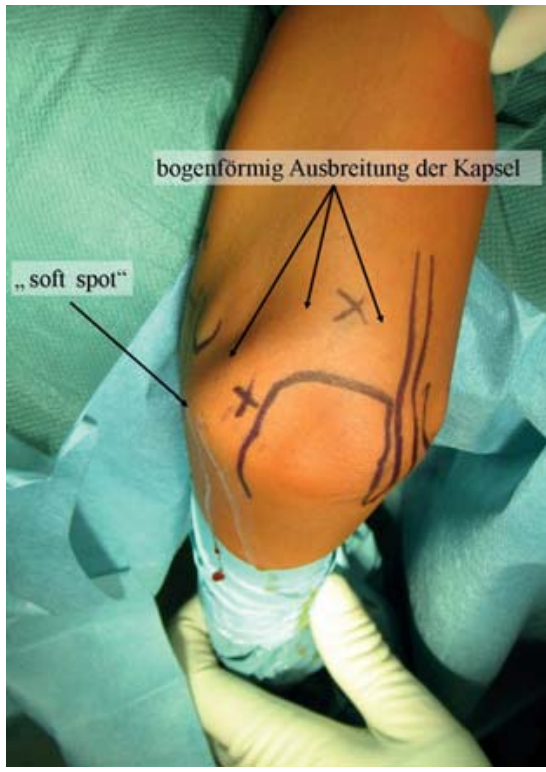

Abb. 3 Auffüllung des Gelenks mit 20-30 m Kochsalz im „soft spot“ führt zu einer bogenförmigen Ausbreitung der Kapsel nach dorsolateral, dabei werden die neurovaskulären Strukturen vom Gelenk in die Peripherie verdrängt.

fernt war, verglichen mit dem weiter distalen Portal bei gleich guter intraartikulärer Übersicht [16]. Der stumpfe Trokar wird Richtung Ellenbogengelenkzentrum gerichtet und von streng radial durch den M. extensor carpi radialis brevis eingebracht.

Das mediale Ellenbogenkompartiment lässt sich durch dieses Portal übersichtlich darstellen. Durch Flexion und Extension lassen sich der Processus coronoideus, die Fossa coronoidea, die Trochlea, die mediale Gelenkkapsel, der mediale Anteil des Caput radii und der vordere Anteil des radioulnaren Gelenks darstellen.

\section{Proximales anteromediales Porta}

Dieses Portal liegt $2 \mathrm{~cm}$ proximal und etwas ventral des medialen Epikondylus, als Variation von Lindenfeld (1 cm proximal und $1 \mathrm{~cm}$ anterior) [17]. Das Portal eignet sich, um das vordere Kompartiment zu betrachten, insbesondere die Bewegung des Radiusköpfchens bei Pround Supination. Beim Zugang muss darauf geachtet werden, ventral des Septum intermusculare mediale einzugehen.

Das Portal wird von einigen Autoren als Anfangsportal empfohlen, insbesondere bei Bauchlage und bei Seitenlage [18] Einige Studien haben gezeigt, dass die Anlage eines superomedialen Portals als erstes Portal einen Vorteil bez. der Si- 


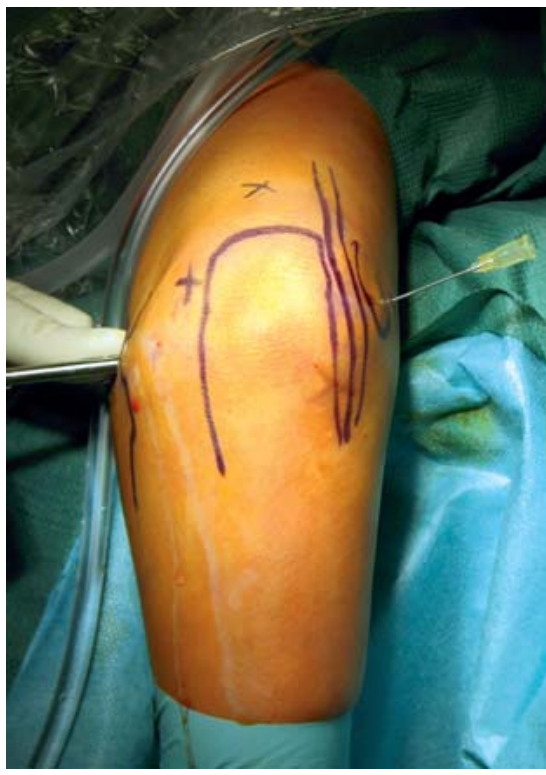

Abb. 4 Das anteromediale Portal wird durch eine Kanülenpunktion unter arthroskopischer Sicht von anterolateral angelegt („outside-in“Technik).

cherheit gibt $[15,17,19]$. Des Weiteren wird die gute Einsehbarkeit in das vordere Kompartiment beschrieben [10].

\section{Anteromediales Portal}

Carson und Andrews beschreiben die Lage $2 \mathrm{~cm}$ distal und $2 \mathrm{~cm}$ ventral des ulnaren Epikondylus [7]. Der Abstand des N. cutaneus antebrachii medialis beträgt nach Lynch et al. $1 \mathrm{~mm}$ und dieser Hautnerv ist bei diesem Portal am stärksten gefährdet [19]. Um den Zugang anzulegen, muss der Ellenbogen $90^{\circ}$ gebeugt werden und das Gelenk aufgefüllt sein, dann beträgt der anteriore Abstand des Portals zum N. medianus 7-14 mm und der Abstand zur A. brachialis im Durchschnitt $17 \mathrm{~mm}$. Ist der Ellenbogen gestreckt, so verläuft der N. medianus direkt im Zugangsweg [16].

Lindenfeld platziert den anteromedialen Zugang weiter proximal und weiter dorsal $(1 \mathrm{~cm}$ proximal und $1 \mathrm{~cm}$ vor dem Epicondylus medialis, ähnlich wie Poehling et al., die das Portal $2 \mathrm{~cm}$ proximal des Epicondylus medialis unmittelbar vor dem Septum intermusculare platzieren) $[9,17]$. Dadurch vergrößert sich der Abstand zum N. medianus auf 22,3 mm, wenn der Zugang nicht rechtwinklig zu den neurovaskulären Strukturen, sondern schräg nach distal in Richtung Ellenbogenzentrum angelegt wird. Der $\mathrm{Zu}$ gang verläuft durch den $\mathrm{M}$. flexor carpi radialis und den $\mathrm{M}$. flexor digitorum su-

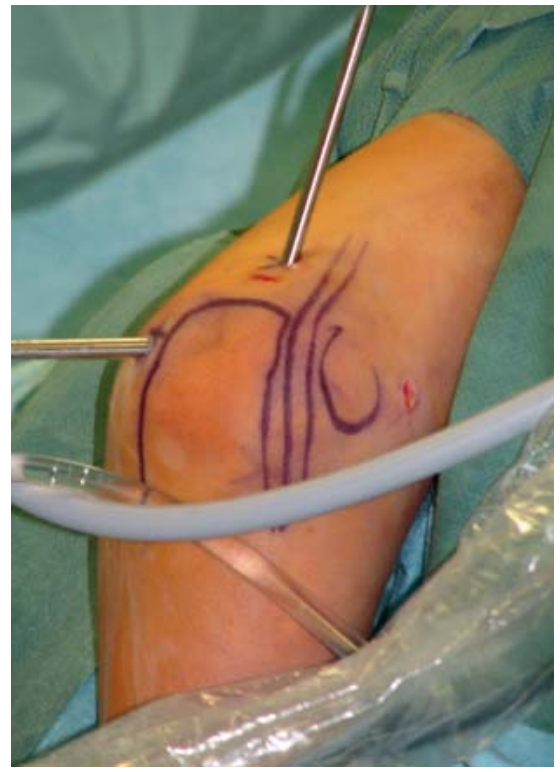

Abb. 5 Arthroskop im posterolateralen Portal und Shaver im posterozentralen transtendinösen Portal.

perficialis und durch Anteile des M. pronator teres.

Das Portal kann durch eine Kanülenpunktion unter arthroskopischer Sicht von anterolateral angelegt werden („outside-in“-Technik), mit dem Vorteil, den Eintrittspunkt gut beurteilen zu können um das Instrumentenmanagement parallel zur Gelenkachse zu optimieren (Abb. 4).

Alternativ hierzu kann das Arthroskop bis an die mediale Kapsel vorgeschoben werden, die Wechselstange kann dann zur Perforation der Kapsel verwendet werden und bis in die Subkutis vorgeschoben werden (,inside-out“-Technik). Durch eine Hautinzision über der Wechselstange kann der Zugang vervollständigt werden.

Über den anteromedialen Zugang lassen sich das Radiusköpfchen, das Capitulum, das Lig. annulare radii, die radiale Fossa, der Processus coronoideus, die Trochlea und die anterolaterale Kapsel darstellen [13].

Bevor das Portal angelegt wird, muss der $\mathrm{N}$. ulnaris palpiert werden, um eine Subluxation nach anterior festzustellen. Dieses Portal ist zu vermeiden, wenn eine Nerventransposition des N. ulnaris vorausgegangen ist. In einem solchen Fall kann präoperativ mit Ultraschall der Nervenverlauf dargestellt werden, um die Anlage des medialen Portals dementsprechend anzupassen. Wenn dies nicht möglich ist, wird die Anlage eines 2$3 \mathrm{~cm}$ großen Zugangs empfohlen, mit einer Präparation auf die Kapsel und Aufsuchen des N. ulnaris. Anschließend wird der Nerv weggehalten und der Zugang erfolgt unter Sicht [5].

\section{Eigenes Vorgehen}

In unserer Klinik beginnen wir nach Auffüllen und Verdrängen des $\mathrm{N}$. radialis mit dem mittleren anterolateralen Portal (ca. $1 \mathrm{~cm}$ ventral und $2 \mathrm{~cm}$ distal des Epicondylus radialis). Wichtig ist, dass nur die Haut inzidiert wird und danach stumpf mit dem Trokar streng von radial in die Kapsel eingegangen wird. Das anteromediale Portal setzen wir in einer Outsidein-Technik, da wir dieses Portal dann genau parallel zu der Gelenkfläche legen können. Dies bringt bei einer durchzuführenden ventralen Kapsulotomie bei einer Kontraktur Vorteile. Der anteromediale Zugang entspricht dann in etwa dem von Carson und Andrews beschriebenen Portal $2 \mathrm{~cm}$ distal und $2 \mathrm{~cm}$ ventral des medialen Epikondylus. Dann wird über Einbringen einer Wechselstange von ulnar die Optik nach ulnar umgesetzt, um die radialen Abschnitte besser betrachten zu können. Der vorher angelegte radiale Zugang wird dann als Arbeitszugang verwendet.

Falls eine schwere Kontraktur mit einer Verengung des Kapselvolumens vorliegt, würden wir empfehlen, primär das anteromediale Potral zum Eingehen in das Gelenk zu verwenden.

\section{Posterolaterales Portal}

Die posteriore Ellenbogenarthroskopie ist im Vergleich zur ventralen sicherer, da die neurovaskulären Strukturen weiter entfernt sind [16]. Das Portal wird 1$3 \mathrm{~cm}$ proximal der Olekranonspitze direkt lateral der Trizepssehne angelegt in Richtung auf die Fossa olecrani (Abb.5). Es können in der Sicht nach proximal die Fossa olecrani und der proximale Anteil des Ellenhakens und nach vorsichtigem Umschwenken nach distal (ohne aus der Kapsel zu rutschen) das dorsale Radiusköpfchen, das proximale Radioulnargelenk und das Capitulum betrachtet werden. Dieser Gelenkabschnitt, das posteriore Humeroradialgelenk, ist eine häufige Lokalisation für freie Gelenkkörper. 


\section{Posterozentrales Portal}

Das Portal wird transtendinös durch die Sehne des M. triceps unter Sicht nach proximal aus dem posterolateralen Portal angelegt und ist zentral in der Fossa olecrani $(1-3 \mathrm{~cm}$ proximal der Olekranonspitze) (Abb.5). Insofern die Mittellinie des dorsalen Oberarms nicht überschritten wird, beträgt der Abstand zum $\mathrm{N}$. ulnaris mindestens $15 \mathrm{~mm}$ und der Abstand zu den Nn. cutanei antebrachii posterior und medialis $25 \mathrm{~mm}[19,20]$. Eine weiter distale Anlage erlaubt die Einsicht in das posteriore radiokapitulare Gelenk.

Das posterozentrale Portal wird in der Regel als Arbeitszugang verwendet, um die Olekranonspitze, die Fossa olecrani und die Triceps-Bursa zu debridieren und von Osteophyten zu befreien. Das posterozentrale Portal kann unter Einsatz einer Wechselstange auch als Sichtportal verwendet werden, um die Fossa olecrani zu inspizieren. Für beide posterioren Portale wird der Trokar bei $90^{\circ}$ gebeugtem Ellenbogen in einem Winkel von ca. $45^{\circ}$ in Richtung auf die Fossa olecrani eingegangen.

\section{Direktes laterales Portal, „soft spot“, unteres dorsolaterales Portal}

Dieses Portal befindet sich ca. $2-3 \mathrm{~cm}$ lateral und distal des posterolateralen Sichtportals im Zentrum des Dreiecks zwischen Radiusköpfchen, Epicondylus lateralis und Olekranon und entspricht dem „soft spot“ (Abb. 3). Der Zugang perforiert den $M$. anconeus und gefährdet keine wichtigen neurovaskulären Strukturen. Der Zugang wird verwendet, um freie Gelenkkörper, eine hypertrophe, schmerzhafte posterolaterale Plica oder einen instabilen Osteochondrosis-dissecans-Herd zu entfernen und/oder debridieren.

\section{Komplikationen}

Mögliche Komplikationen bei der Ellenbogenarthroskopie schließen den oberflächlichen und tiefen Infekt, eine persistierende Fistelung, das Auftreten eines Kompartment-Syndroms und das Auftreten von vorübergehenden und persistierenden Gefäß- und Nervenläsionen ein $[8,19,21-27]$. Insbesondere die arthroskopische Arthrolyse geht mit dem Risiko der Verletzungen der peripheren Nerven, Gefäße und des Knorpels einher [28]. Die gefürchtetste Komplikation bei der Ellenbogenarthroskopie ist die Ner- venverletzung. Es gibt Berichte über Nervenläsionen von jedem den Ellenbogen kreuzenden Nerv, in unterschiedlicher Ausprägung $[24,26,29,30]$. Die meisten Nervenläsionen sind vorübergehend, aber auch persistierende Nervenverletzungen wurden beschreiben [23].

Neben einer direkten Verletzung mit dem Skalpell bei der Hautinzision kann es zu einer Verletzung durch den stumpfen Trokar oder durch scharfe Instrumente wie den Shaver oder Punch kommen. Es kann auch zu einem versehentlichen Aufwickeln eines Nervs durch ein rotierendes Instrument kommen [7,8,26, 29-32].

Die meisten Nervenläsionen sind durch Schwellungszustände, Druckschädigungen durch Trokare oder durch Dehnungsschäden bei postoperativer intensivierter Bewegung verursacht. Diese Schäden sind glücklicherweise meist vorübergehend [7, 19,33].

Eine umfangreiche Nachuntersuchung von 473 Arthroskopien bei 449 Patienten in einem Zeitraum von 18 Jahren von Kelly et al. zeigte eine Komplikationsrate von $10 \%$, die wesentlich höher ist als die Komplikationsrate von $1-2 \%$ bei Knieoder Schulterarthroskopien [34].

Es konnten Risikofaktoren identifiziert werden, die eine zeitweise Nervenschädigung begünstigen, hierzu gehören die Diagnose einer rheumatoiden Arthritis und die Flexionskontraktur des Ellenbogens. Bei der rheumatoiden Arthritis besteht meist eine ausgeprägte Synovialitis, die die Orientierung erschwert und eine Synovektomie notwendig macht. Des Weiteren kann die knöcherne Anatomie aufgrund von Erosionen verändert sein [5].

Tiefe Gelenkinfekte nach Ellenbogenarthroskopie sind selten und werden mit ca. $0,8 \%$ (4 von 473 Ellenbogen) angegeben. Oberflächliche Infektionen und Fisteln werden zu ca. 7\% nach Ellenbogenarthroskopie beschrieben [34].

Eine seltene Komplikation ist das Auftreten eines Hautemphysems nach Ellenbogenarthroskopie der oberen Extremität durch einen Ventilmechanismus über die Haut bei der postoperativen, intensiven Mobilisiation des Ellenbogens. Eine zügige Differenzierung zum Ausschluss einer Gasbrandinfektion ist notwendig, um eine adäquate Behandlung einleiten zu können [35].

\section{Fazit für die Praxis}

Die Indikationen zur Ellenbogenarthroskopie beinhalten die diagnostische $\mathrm{Ar}$ throskopie, die Entfernung freier Gelenkkörper, die Osteophytenabtragung, die Synovektomie, Behandlung der Osteochondrosis dissecans und die Arthrolyse bei Kontrakturen des Ellenbogens. Die genaue Kenntnis der Anatomie ist die Voraussetzung, um die Komplikationsrate aufgrund der engen Lagebeziehung zwischen Kapsel und neurovaskulären Strukturen gering zu halten. Eine standardisierte Lagerung, das Auffüllen des Gelenks vor der Zugangsanlage, sowie die stumpfe Präparation nach Hautdurchtrennung sind wichtige Bausteine für eine komplikationslose Operation.

Häufige Portalanlagefehlversuche und das häufige Wechseln der Portale führen rasch zu Flüssigkeitsansammlungen im Subkutangewebe und zu einer Kompression der Gelenkkapsel. Dadurch wird die Fortsetzung der Arthroskopie erschwert.

Die Ellenbogenarthroskopie hat im Vergleich zur Kniegelenkarthroskopie eine relativ flachere Lernkurve und sollte von Operateuren durchgeführt werden, die schon arthroskopische Erfahrung an größeren Gelenken gesammelt haben.

\section{Literatur}

${ }^{1}$ Morrey BF, Askew LJ, Chao EY. A biomechanical study of normal functional elbow motion. J Bone Joint Surg [Am] 1981; 63: 872-877

2 Barthel T, Rolf O. Ellenbogenarthroskopie. Arthroskopie 2006; 19: 298-303

3 Simmelbauer B, Habermeyer P. [Standardized examination of the elbow]. Unfallchirurg 1996; 99: 548-554

${ }^{4}$ Ahmad CS, Vitale MA. Elbow arthroscopy: setup, portal placement, and simple procedures. Instr Course Lect 2011; 60: 171-180

5 Steinmann SP. Elbow arthroscopy: where are we now? Arthroscopy 2007; 23: 1231-1236

${ }^{6}$ O'Driscoll SW, Lawton RL, Smith AM. The "moving valgus stress test" for medial collateral ligament tears of the elbow. Am J Sports Med 2005; 33: 231-239

7 Andrews JR, Carson WG. Arthroscopy of the elbow. Arthroscopy 1985; 1: 97-107

8 O'Driscoll SW, Morrey BF. Arthroscopy of the elbow. Diagnostic and therapeutic benefits and hazards. J Bone Joint Surg [Am] 1992; 74: 84-94

${ }^{9}$ Poehling GG, Whipple TL, Sisco L et al. Elbow arthroscopy: a new technique. Arthroscopy 1989; 5: 222-224

10 Stothers K, Day B, Regan WR. Arthroscopy of the elbow: anatomy, portal sites, and a description of the proximal lateral portal. Arthroscopy 1995; 11: 449-457

11 Gallay SH, Richards RR, O'Driscoll SW. Intraarticular capacity and compliance of stiff and normal elbows. Arthroscopy 1993; 9: 9-13

12 Unlu MC, Kesmezacar H, Akgun I et al. Anatomic relationship between elbow arthroscopy portals and neurovascular structures in 
different elbow and forearm positions. J Shoulder Elbow Surg 2006; 15: 457-462

13 Porcellini G, Paladini P, Campi F et al. Arthroscopic neurolysis of the ulnar nerve at the elbow. Chir Organi Mov 2005; 90: 191-200

14 Field LD, Altchek DW, Warren RF et al. Arthroscopic anatomy of the lateral elbow: a comparison of three portals. Arthroscopy 1994; 10: 602-607

15 Verhaar J, van Mameren H, Brandsma A. Risks of neurovascular injury in elbow arthroscopy: starting anteromedially or anterolaterally? Arthroscopy 1991; 7: 287-290

${ }^{16}$ Noonburg GE, Baker jr. CL. Elbow arthroscopy. Instr Course Lect 2006; 55: 87-93

17 Lindenfeld TN. Medial approach in elbow arthroscopy. Am J Sports Med 1990; 18: 413 417

18 Plancher KD, Bishai SK. Basic of elbow arthroscopy: setup, portals, and technique. Techniques in Orthopaedics 2006; 21: 239-249

${ }^{19}$ Lynch GJ, Meyers JF, Whipple TL et al. Neurovascular anatomy and elbow arthroscopy: inherent risks. Arthroscopy 1986; 2: 190-197

20 Baker jr. CL, Jones GL. Arthroscopy of the elbow. Am J Sports Med 1999; 27: 251-264

${ }^{21}$ Angelo RL. Advances in elbow arthroscopy. Orthopedics 1993; 16: 1037-1046

22 Jerosch J, Castro WH. [Arthroscopy of the elbow joint. Long-term results, complications and indications.] Unfallchirurg 1992; 95: 405-411
23 Jones GS, Savoie III FH. Arthroscopic capsular release of flexion contractures (arthrofibrosis) of the elbow. Arthroscopy 1993; 9: 277283

24 Papilion JD, Neff RS, Shall LM. Compression neuropathy of the radial nerve as a complication of elbow arthroscopy: a case report and review of the literature. Arthroscopy 1988; 4: $284-286$

${ }^{25}$ Redden JF, Stanley D. Arthroscopic fenestration of the olecranon fossa in the treatment of osteoarthritis of the elbow. Arthroscopy 1993; 9: 14-16

${ }^{26}$ Ruch DS, Poehling GG. Anterior interosseus nerve injury following elbow arthroscopy. Arthroscopy 1997; 13: 756-758

${ }^{27}$ Rupp S, Tempelhof S. Arthroscopic surgery of the elbow. Therapeutic benefits and hazards. Clin Orthop Relat Res 1995; 313: 140-145.

28 Van Zeeland NL, Yamaguchi K. Arthroscopic capsular release of the elbow. J Shoulder Elbow Surg 2010; 19: 13-19

${ }^{29}$ Dumonski ML, Arciero RA, Mazzocca AD. Ulnar nerve palsy after elbow arthroscopy. Arthroscopy 2006; 22: 577.e1-3

${ }^{30}$ Haapaniemi T, Berggren M, Adolfsson L. Complete transection of the median and radial nerves during arthroscopic release of posttraumatic elbow contracture. Arthroscopy 1999; 15: 784-787

${ }^{31}$ Hahn M, Grossman JA. Ulnar nerve laceration as a result of elbow arthroscopy. J Hand Surg Br 1998; 23: 109
${ }^{32}$ Thomas MA, Fast A, Shapiro D. Radial nerve damage as a complication of elbow arthroscopy. Clin Orthop Relat Res 1987; 215: 130 131

33 Poehling GG, Ekman EF. Arthroscopy of the elbow. Instr Course Lect 1995; 44: 217-223

34 Kelly EW, Morrey BF, O'Driscoll SW. Complications of elbow arthroscopy. J Bone Joint Surg [Am] 2001; 83: 25-34

${ }^{35}$ Dexel J, Schneiders W, Kasten P. Subcutaneous emphysema of the upper extremity after elbow arthroscopy. Arthroscopy 2011; 27: 1014-1017

\section{Dr. med. Julian Dexel}

Facharzt

Prof. Dr. med. Philip Kasten

Oberarzt

Sektion Schulter und Ellenbogenchirurgie und Sportorthopädie Klinik und Poliklinik für Orthopädie des Universitätsklinikums

Carl Gustav Carus Dresden Fetscherstraße 74

01307 Dresden

philip.kasten@uniklinikum-dresden.de 